\title{
SPECTRAL ASSOCIATION OF THE 7 AUGUST 1972 SOLAR \\ RADIO BURST WITH PARTICLE ACCELERATION
}

\author{
J. P. CASTELLI and A. L. CARRIGAN \\ Air Force Cambridge Research Laboratories, Bedford, Mass., U.S.A. \\ and \\ H. C. $\mathrm{KO}$ \\ Ohio State University, Columbus, Ohio, U.S.A.
}

\begin{abstract}
Solar Phys.). Microwave burst data from the August 7, 1972 event recorded on nine discrete frequencies between 245 and $35000 \mathrm{MHz}$ at the Sagamore Hill Radio Observatory (Figure 1) provide a basis for correlation studies (especially timing in-
\end{abstract}

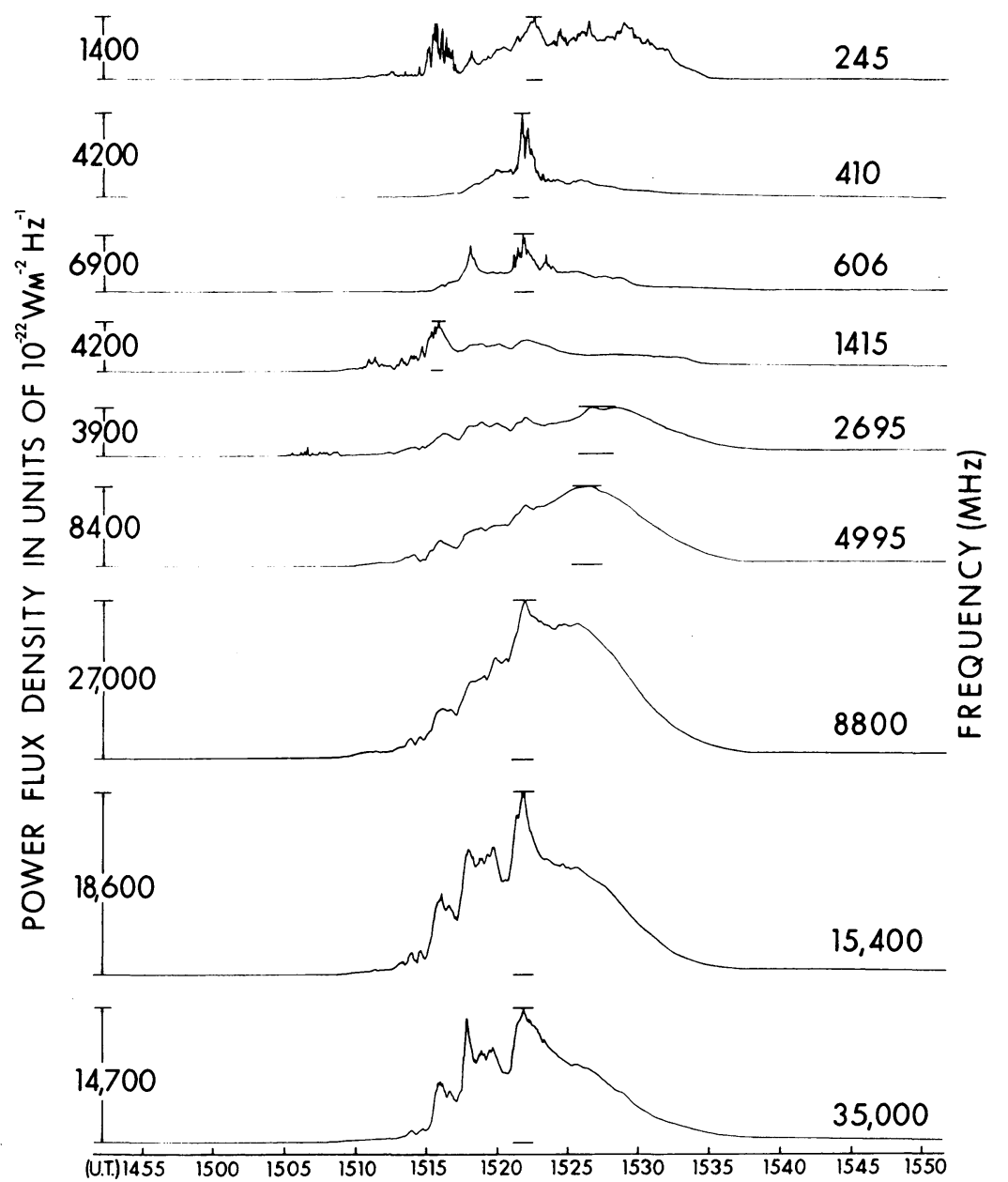

Fig. 1. Great burst observed 7 August, 1972 at Sagamore Hill Radio Observatory, Hamilton, Mass. 
formation) of the associated white light flare, the high energy particle emission, type II bursts, and many other phenomena. This is perhaps the first time that sufficient radio coverage (i.e., data above $10000 \mathrm{MHz}$ ) was available to obtain the spectral slope information (related to electron-energy distribution) which is inherent in this part of the spectrum. Heretofóre, timing was related to burst flux density profile variations. Improved correlations resulted from shorter centimeter wavelength data which supplied more accurate timing information than that derived from $\mathrm{H} \alpha$ observations. The shape and intensity of the burst peak flux density spectrum has also been used for qualitative analysis of energetic particle and white light events. The ultimate good may possibly come from spectral analysis of the minute by minute variation of the burst microwave radiation spectral slope $\alpha$ (above $f_{\max }$ ) in the area between 15000 and $35000 \mathrm{MHz}$. This may be used alone or in relation to the position of $f_{\max }$, where $f_{\max }$ is the frequency of burst maximum emission at a given time. This is basically our present investigation.

It is well known that the synchrotron radiation from an ensemble of highly relativistic electrons with the power-law energy distribution $N(E) \propto E^{-\gamma}$ has the radiated

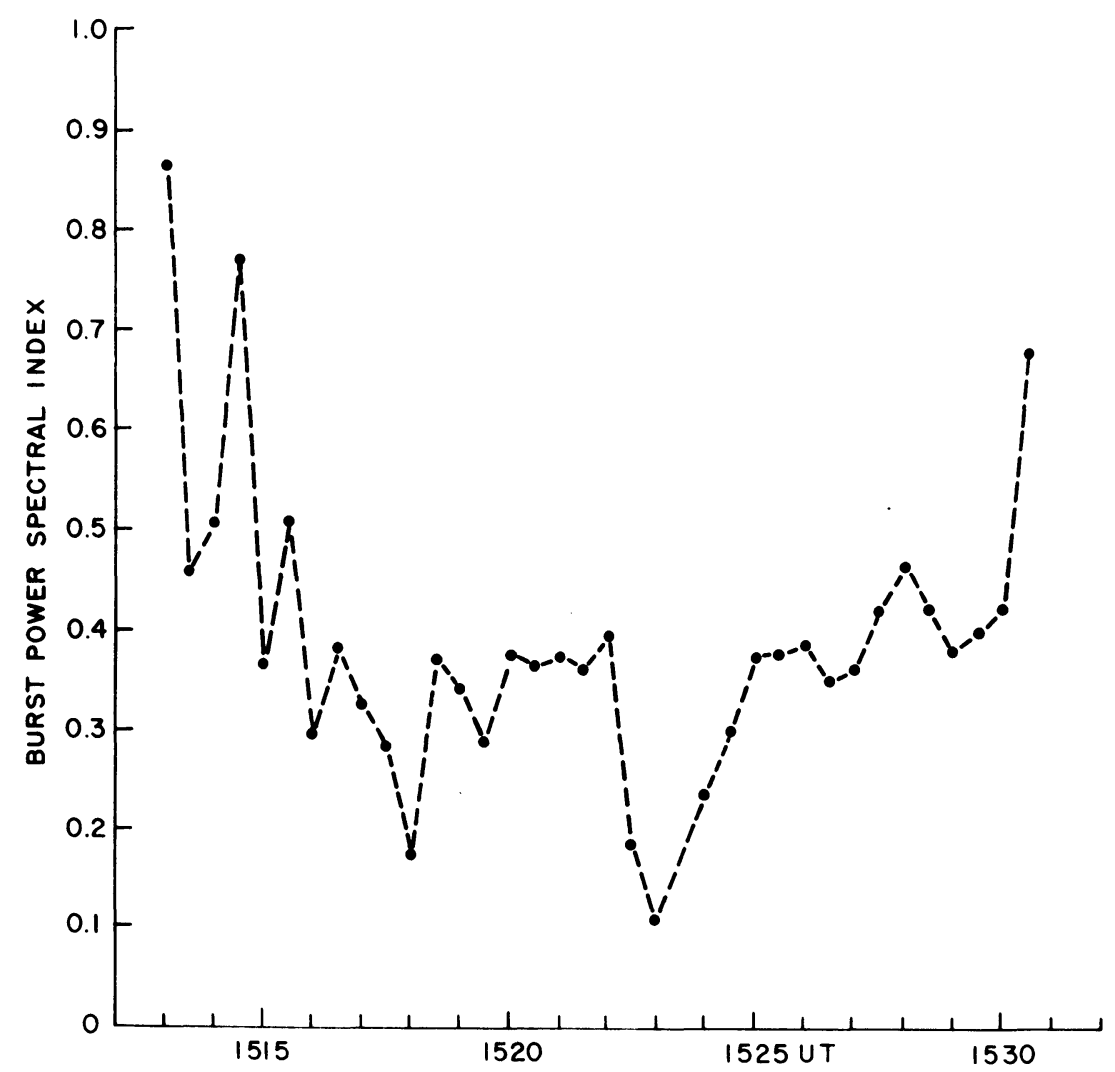

Fig. 2. Burst power spectral index variation during principal part of event of 7 August 1972, based on measured data at 15000 and $35000 \mathrm{MHz}$. 
power spectrum given by $f^{-\alpha}$ where $\alpha=(\gamma-1) / 2(\gamma$ and $\alpha$ are the electron-energy spectral index and the microwave radiation power spectral index, respectively). Unfortunately, this simple relation is not directly applicable to microwave solar bursts since the low and medium energy electrons play dominant roles (gyro-synchrotron emission) instead of ultrarelativistic electrons. Still, there is a strong tendency with very energetic events for this $\alpha=(\gamma-1) / 2$ relation to apply, especially as we go further into the millimeter range. This is evidenced by the increasing flatness (hardness) of the radiation slopes above $f_{\max }$. To evaluate the functional relation between $\alpha$ and $\gamma$, one has to integrate the general synchrotron radiation formulas with respect to the powerlaw energy distribution of electrons. By means of a computer program, we have calculated the volume emissivity as a function of frequency for various values of $\gamma$ and $\theta$, where the latter is the angle of observation measured from the direction of the magnetic field. The spectral index is found to vary with frequency. From these numerical data we have obtained a set of curves relating $\alpha$ and $f / f_{H}\left(f_{H}\right.$ is the gyrofrequency) for various $\gamma$ and $\theta$. From these curves, the electron-energy spectral index $\gamma$ can be estimated from an assumption of $\theta$ and a given set of $\alpha$ and $f / f_{H}$.

For the August 7, 1972 event during the most intense part of the burst, the microwave radiation spectral index varies between $\alpha=0.2$ and 1.0 (see Figure 2). The corresponding electron-energy spectral index is estimated at between $\gamma=1.2$ and 2.0. Thus, the distribution of electrons during this period had an energy spectral index as hard as 1.2 (1.6 on an average). The $\alpha$ variation $(0.2$ to 1.0$)$ over about $15 \mathrm{~min}$ is found to be very much flatter than for non-white lights flares and non-ground level events. In relating the white light flare development on August 7, 1972 to burst spectral hardness, an $\alpha$ of approximately 0.2 is apparent at the white light flare start near $1518 \mathrm{UT}$. Its maximum near 1523 UT agrees well with the time of burst maximum spectral hardness. The white light flare seemed to persist until about 1530 UT at which time the burst $\alpha$ rose abruptly above 0.4 . These burst spectral data are correlated only with timing aspects of the flare phenomena at present. As such, they serve as a signature. This study does not address the problem of whether the white light flare was produced by high or low energy electrons or protons, all of which were abundant during the period.

While the radio burst spectrum was varying in hardness, the frequency of maximum radio emission was also varying; the position of $f_{\max }$ reached its highest excursion at about 1518 UT (Figure 3). This tends to suggest that the greatest influx of electrons occurred at this time. If the emission is primarily from non-thermal electrons, then an increase in the average electron-energy (injected into the microwave source) would shift the position of $f_{\max }$ upward. An increase in the influx of thermal electrons contributing to ionized-medium suppression would also effectively shift $f_{\max }$ upward. In either case, an increase in the magnetic field intensity is not required.

There are several points which are still incomplete. We have not investigated sufficiently the total number of electrons nor the total amount of energy involved. A preliminary calculation of the radio burst energy transformed into radiation in the $600-35000 \mathrm{MHz}$ range yields $\sim 0.5 \times 10^{26}$ ergs. Since the same electrons may be re- 


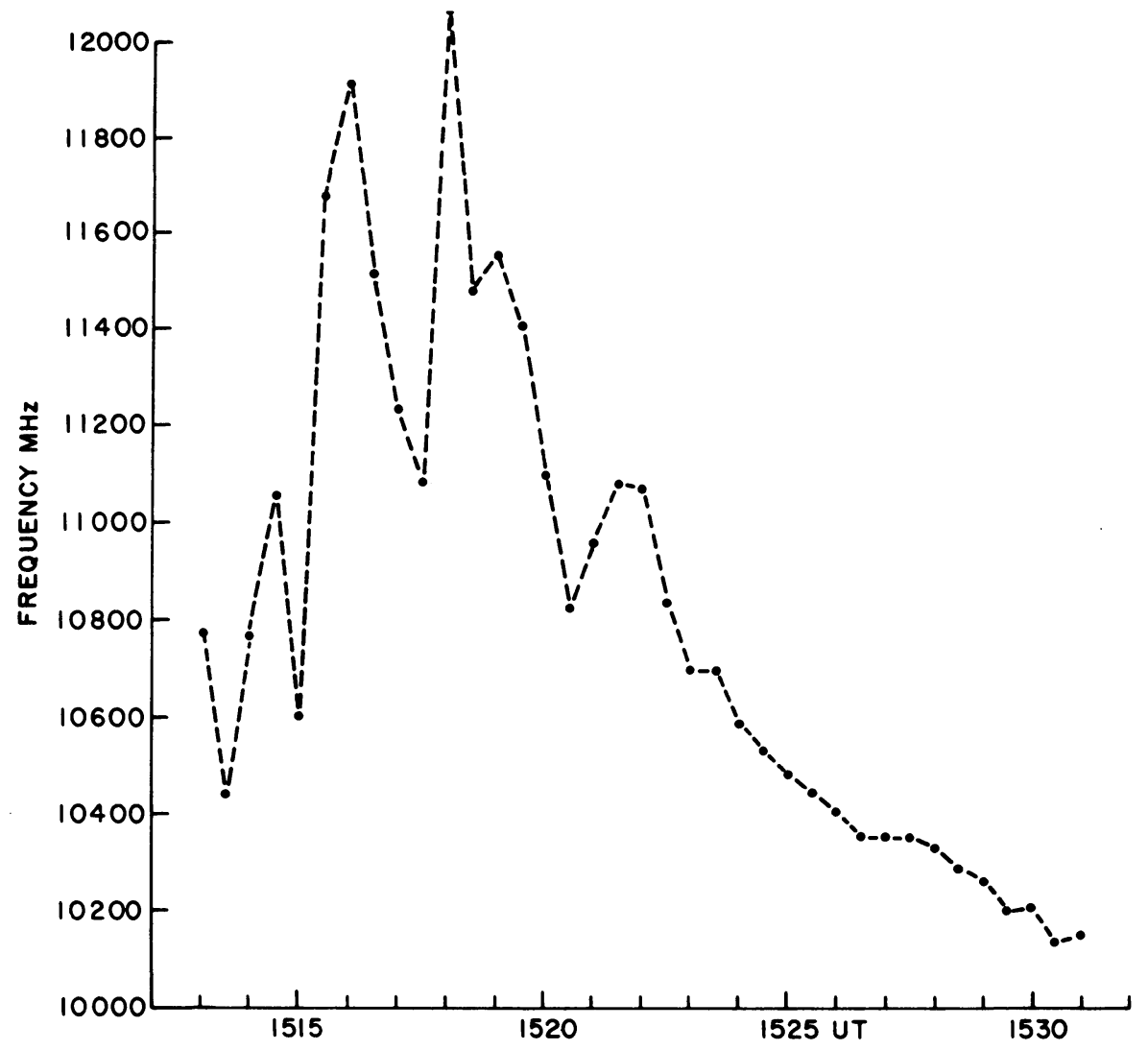

Fig. 3. Variation of centimeter wavelength burst spectral maximum $\left(f_{\max }\right)$, during principal part of event of 7 August 1972. $f_{\max }$ was determined from best fit quadratic to measured flux densities at 4995,8800 and $15400 \mathrm{MHz}$.

sponsible for hard X-rays, we will investigate whether the energy spectrum derived in our study is consistent with the hard X-ray spectrum. (For this purpose we have to choose either the thin or thick target interpretation of the X-ray spectrum which will give considerably different results.) Hard X-ray data are now available from which to complete the study. 